\title{
Comparative study of hierarchically structured and bifunctional core-shell rare earth nanoparticles
}

\section{Amanda J. Morais* (IC), Flávia S. Ferreira (PG), Italo O. Mazali (PQ), Fernando A. Sigoli (PQ)}

\begin{abstract}
Multifunctional systems based on lanthanide-doped nanoparticles can combine optical and magnetic properties with wide applications in diagnosis and treatment of diseases. Hierarchically nanostructure core@shell systems of NaGdF4:Er:Yb, NaGdF4:Er:Yb@NaYF4:Yb@NaYF4:Tb:Yb and NaYF4:Tb:Yb@NaYF4:Yb@NaGdF4:Er:Yb composition were synthesized to investigate the magnetic and optical properties in order to obtain nanoparticles with morphology, size and properties that allow the application in biological media. Information on the crystalline phase, particle morphologies and the upconversion emission spectra were obtained using: X-Ray Diffraction (XRD), Transmission Electron Microscopy (TEM) and Photoluminescence Spectroscopy.
\end{abstract}

Key words:

Nanoparticles, upconversion, rare earth

\section{Introduction}

Lanthanides ions-doped nanoparticles have attracted the attention of the scientific community due their magnetic and luminescent properties favorable to obtain multifunctional systems that can be applied in the diagnosis and treatment of diseases. ${ }^{1,2}$ These materials exhibit resistance to photochemical degradation and fine and well-defined emission bands. One of the phenomena responsible for the development of this systems is the possibility of upconversion emission (UC) ${ }^{3,4}$ This phenomenon is a nonlinear effect where multiple lower energy excitation photons produce higher emission photons. ${ }^{3,4}$ Hence, those systems are promising for cell imaging and photodynamic therapy. ${ }^{3}$ Associated with their luminescent characteristics, paramagnetic lanthanides are a promising class of contrast agents in Magnetic Resonance Imaging (MRI). ${ }^{2}$ Thus, this work involves the investigation of the optical and magnetic properties of systems synthesized in order to obtain nanoparticles that present morphology, size and properties that allow the application in biological media.

\section{Results and Discussion}

The methodology used for the synthesis of

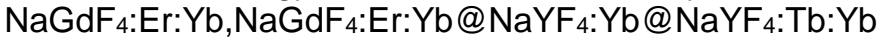
and $\mathrm{NaYF}_{4}: \mathrm{Tb}: \mathrm{Yb} @ \mathrm{NaYF}_{4}: \mathrm{Yb} @ N a G d F_{4}: \mathrm{Er}: \mathrm{Yb}$ was the thermal decomposition of metal precursors in solvents with high boiling points. ${ }^{5}$ The trifluoracetates of rare earth $\left.\left[\mathrm{TROOCCF}_{3}\right)_{3}\right]$ and sodium $\left[\mathrm{NaOOCCF}_{3}\right]$ were used as precursors. The solvents and stabilizers of the synthesis were oleic acid, oleylamine and 1-octadecene. The control temperature, reaction time and addition of the precursor resulted into the cubic ( $\alpha$ ) or hexagonal $(\beta)$ crystalline phases. Transmission Electron Microscopy (TEM) and X-Ray Powder Diffraction (XRD) showed the formation of particles of uniform size and morphology with well-defined crystalline phases. Emission spectra under $980 \mathrm{~nm}$ excitation exhibit the typical emission lines of erbium(III) ion. Furthermore, the results obtained through Photoluminescence Spectroscopy have suggested that the hexagonal phase is more appropriate to improve the optical properties of the system. After modifications in the synthesis method a sample was obtained which, besides showing high emission intensities at very low power, allowed the excitation at one more wavelength (980 and
$808 \mathrm{~nm}$ ), showing the potential of nanoparticles biological applications.

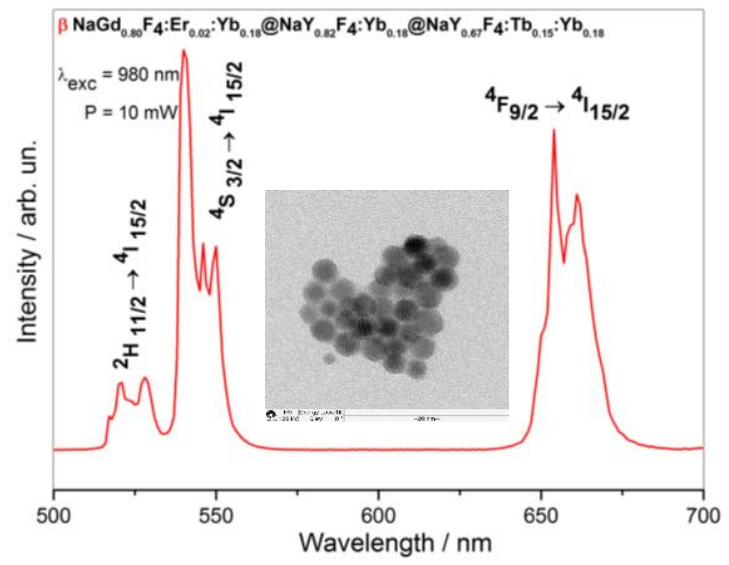

Image 1. Upconversion emission and Transmission Electron Microscopy image of $\beta$ $\mathrm{NaGd}_{0,80} \mathrm{~F}_{4}: \mathrm{Er}_{0,02}: \mathrm{Yb}_{0,18} @ \mathrm{NaY}_{0,82} \mathrm{~F}_{4}: \mathrm{Yb}_{0,18} @ \mathrm{NaY}_{0,67} \mathrm{~F}_{4}: \mathrm{Tb}_{0,15}:$ $\mathrm{Yb}_{0,18}$ nanoparticles.

\section{Conclusions}

The system under study presented satisfactory results that allowed the exploration of several parameters and the verification their influence on the displayed properties. The characterizations performed have suggested that the synthesis method provides many modifying factors than can be better investigated. Lastly, all the obtained results show the synthesized lanthanide-doped systems potential as a promising class of biological probes.

\section{Acknowledgement}

FAPESP, CNPq, CAPES, INOMAT and Multi-user Laboratory for Advanced Optical Spectroscopy.

${ }^{1}$ Gautam, A., et al., Journal of Material Chemistry, 2013, 1, 5186-5200.

${ }^{2}$ Wu, L., et al., Chem. Rev, 2016, 116, 10473-512.

${ }^{3}$ Nadort, Annemarie, et al., Nanoscale, 2016, 8, 13099-13130.

${ }^{4}$ Dong, H., et al., Chem. Rev. 2015, 115, 10725-815.

5 Johnson, N. J. J., et al., Nano Research, 2013,6, 547-561. 